Marta Tonello, Elisa Bison, Maria Grazia Cattini, Elena Pontara, Luca laccarino, Gentian Denas, Chunyan Cheng and Vittorio Pengo*

\title{
Anti-phosphatidyl-serine/prothrombin antibodies (aPS/PT) in isolated lupus anticoagulant (LA): is their presence linked to dual test positivity?
}

https://doi.org/10.1515/cclm-2021-0692

Received June 13, 2021; accepted August 8, 2021;

published online August 16, 2021

\section{Abstract}

Objectives: Anti phosphatidylserine/prothrombin antibodies (aPS/PT) are often present in patients with antiphospholipid syndrome (APS) and might be relevant in the pathogenesis of this condition. They are major determinant of lupus anticoagulant (LA) in triple-positive antiphospholipid (aPL) profile. Whether they are present and pathogenic in patients with isolated LA [negative anticardiolipin (aCL) and anti $\beta 2$-glycoprotein I (aß2GPI) antibodies] is a matter of debate.

Methods: We measured aPS/PT in a large number of isolated LA with the aim to ascertain whether there is a link between the way isolated LA is assessed and the presence of these antibodies. APS/PT were measured in 86 patients with isolated LA (aCL- and abeta2GPI-). LA was assessed by two test systems, the dilute Russell Viper Venom Time (dRVVT) and the Silica Clotting Time (SCT).

Results: Sixty-six (77\%) individuals with isolated LA were positive for aPS/PT (IgM 44, IgG and IgM 15, IgG in 7). Diagnosis of LA was made based on positive results in both dRVVT and SCT in 40 patients (Group 1) and based on only one positive test in the remaining 46 patients (Group 2). The rate of positive aPS/PT antibodies was significantly higher

\footnotetext{
${ }^{*}$ Corresponding author: Prof. Vittorio Pengo, MD, Thrombosis Research Laboratory, University of Padua, Campus Biomedico, 'Pietro d'Abano', Via Orus 2/B, 35129, Padua, Italy; and Arianna Foundation on Anticoagulation, Bologna, Italy, Phone: +39 0498212377 , E-mail: vittorio.pengo@unipd.it. https://orcid.org/0000-0003-20646071

Marta Tonello and Luca laccarino, Department of Medicine, Rheumatology Section, University of Padua, Padua, Italy Elisa Bison, Maria Grazia Cattini, Elena Pontara, Gentian Denas and Chunyan Cheng, Thrombosis Research Laboratory, University of Padua, Padua, Italy
}

in Group 1 (OR=7.2, 95\% CI 1.9-27.0, $\mathrm{p}<0.002)$. Moreover, the titre of IgM aPS/PT was significantly increased in Group 1 as compared to Group 2 (137 U, IQR 64-179 vs. 43 U, IQR 11-120, $\mathrm{p}=0.008$ ).

Conclusions: These data indicate an association between LA based on two positive coagulation tests and the presence of aPS/PT antibodies, especially of IgM isotype.

Keywords: antibodies; lupus anticoagulant; phosphatidylserine; prothrombin.

\section{Introduction}

Lupus anticoagulant (LA) is considered the main risk factor for thrombosis in patients with antiphospholipid antibodies (aPL) [1]. However, there are two distinct types of LA, one associated and the other not associated (isolated LA) with the presence of anti cardiolipin (aCL) and anti- $\beta 2$-glycoprotein I antibodies (aß2GPI) [2]. Anti phosphatidylserine/prothrombin complex antibodies (aPS/PT) were present in both types of LA when both the dilute Russell Viper Venom Time (dRVVT) and silica clotting time (SCT) were positive. Apparently, the IgM isotype of aPS/PT is more common in isolated LA while the IgG isotype prevails in triple-positive patients. The relative risk for thrombosis linked to the different types of LA is a matter of debate. In our hands, a longitudinal cohort study of isolated LA showed a small number of thromboembolic events in the follow-up period [3]. On the other hand, a recent cross sectional study found isolated LA to be strongly associated with vascular thrombosis [4]. APS/PT are the major determinant of LA activity and might substitute LA assays when its determination is impaired by the presence of anticoagulants in the tested plasma [5, 6]. For this reason, we extended the aPS/PT determination to a large number of isolated LA with the aim to ascertain whether there is a link between the way isolated LA is diagnosed and the presence of aPS/PT antibodies. 


\section{Materials and methods}

This is a cross sectional study including 436 non-anticoagulated LA positive patients referred for thrombophilia screening to Padua thrombosis centre and the Rheumatology outpatient clinic of the University of Padua Medical Centre in the last 10 years. Of these, 86 were isolated LA (negative for aCL and aß2GPI antibodies). LA were assessed by two test systems of differing analytical principles, dRVVT and SCT assays (HemosIL dRVVT and HemosIL SCT, Werfen Group, Milan, Italy), following the international guideline for LA testing [7]. Patients were considered as having a strong LA when the dRVVT and SCT screening, mixing and confirm tests were positive and weak when only one test was positive [8]. The thrombin time test to exclude the presence of heparin in tested plasma was within the normal range. Home-made ELISA methods were used, as described elsewhere [9], for the detection of IgG/IgM aß2GPI and IgG/IgM anti-cardiolipin (aCL) antibodies. Data were then confirmed using QUANTA Flash aCL IgG/ IgM and QUANTA Flash $\beta 2$ GPI IgG/IgM (INOVA Diagnostican San Diego, CA, USA). Patient's laboratory data were confirmed after 12 weeks. After obtaining the written consent from each patient, residual plasma was stored at $-80^{\circ} \mathrm{C}$ for further studies. IgG and IgM aPS/PT antibodies were measured by ELISA (QUANTA Lite, Werfen Group, Milan, Italy). All the ELISA assays were performed following the guidance from the Scientific Standardization Committee on lupus anticoagulant/Antiphospholipid Antibodies of the International Society of Thrombosis and Haemostasis [10]. The institutional review board for observational studies and the Audit Committee of University of Padua Medical Centre approved the study design. The study was conducted in accordance with the principles of the Declaration of Helsinki.

\section{Statistics}

Descriptive statistics are reported as appropriate: continuous data are reported as mean and standard deviation or median and interquartile range for data without normal distribution. The Easy Fischer Exact test was used at univariate analysis to evaluate any associations between the categorical variables. Mann-Whitney test was used to compare values without a normal distribution. Nonparametric Spearman test was used to correlate dRVVT and SCT ratios with values of IgM aPS/PT antibodies. GRAPHPAD-INSTAT 3 (GraphPad Software) was used for the statistical analysis (www.graph pad.com). A p-value $<0.05$ value was considered statistically significant.

\section{Results}

We collected the plasma of 86 LA positive patients that were negative for aCL and aß2GPI confirmed by in-house ELISA and commercial kits (isolated LA) and their characteristics are shown in Table 1 . Mean age was 40 years and $62(72 \%)$ were females. Reasons for testing were the presence of clinical criteria for APS in 27 cases while for the remaining 59 checking for LA was determined by the presence of non-criteria APS or by the presence of an autoimmune disease. Diagnosis of LA was made based on positive results in both dRVVT and SCT in 40 patients (Group 1) and based on only one positive test in the remaining 46 patients (Group 2).

As shown in Figure 1, of 86 patients with isolated LA, 66 (77\%) were positive for aPS/PT: these antibodies were of IgM isotype in 44 cases, IgG and IgM in 15 and positive for IgG isotype in 7 cases. As shown in Figure 2, when both LA tests were positive (Group 1), aPS/PT antibodies were present in 37 out of 40 cases (92\%) while 29 of 46 were positive for aPS/PT in Group 2 (OR=7.2, 95\% CI 1.9-27.0, $\mathrm{p}<0.002)$. In the 37 aPS/PT positive cases in Group 1, the isotype was IgM in 27 cases, IgM and IgG in 6 and IgG in 4 cases; in the $29 \mathrm{aPS} / \mathrm{PT}$ positive cases in Group 2, the isotype was IgM in 17 cases, IgM and IgG in 8 and IgG in 4 cases (Figure 3). As far as IgM titre is concerned (Figure 4), median IgM values in Group 1 were significantly higher than those in Group 2 (137 U, IQR 64-179 vs. 43 U, IQR 11-120, p=0.008). Median IgG aPS/PT titre in Group 1 was not statistically different of those in Group 2 (11 U, IQR 6-31 vs. 7 U, IQR 5-35, p=0.3, Figure 5).

\section{Discussion}

In this study, around a half of isolated LA were diagnosed based on positivity in both dRVVT and SCT assays. This figure is slightly different from our previous series of LA

Table 1: Characteristics of patients with isolated lupus anticoagulant.

\begin{tabular}{lr}
\hline $\mathrm{n}$ & 86 \\
Age, years ${ }^{\mathrm{a}}$ & $40 \pm 24$ \\
Sex, M/F & $24 / 62$ \\
Reasons for testing, $\mathrm{n}$ & \\
- Previous venous or arterial thromboembolism & 23 \\
- Undifferentiated connective tissue disease & 11 \\
- Thrombophilia screening & 9 \\
- Psoriatic arthritis & 7 \\
- Systemic Lupus erythematosus & 5 \\
- Pregnancy morbidity & 4 \\
- Sjögren's syndrome & 3 \\
- Systemic sclerosis & 2 \\
- Thrombocytopenia & 2 \\
- Miscellanea autoimmune diseases & 4 \\
- Unknown reasons & 16 \\
Laboratory data & \\
Coagulation tests & \\
Two positive test (dRVVT and SCT), $\mathrm{n}(\%)$ & 15 \\
One test positive, $\mathrm{n}$ (\%) & 31 \\
dRVVT, $\mathrm{n}$ & $447)$ \\
SCT, n & $46(53)$ \\
\hline
\end{tabular}

${ }^{a}$ Data are expressed a mean \pm SD. dRVVT, Russell Viper Venom Time; SCT, Silica Clotting Time. 


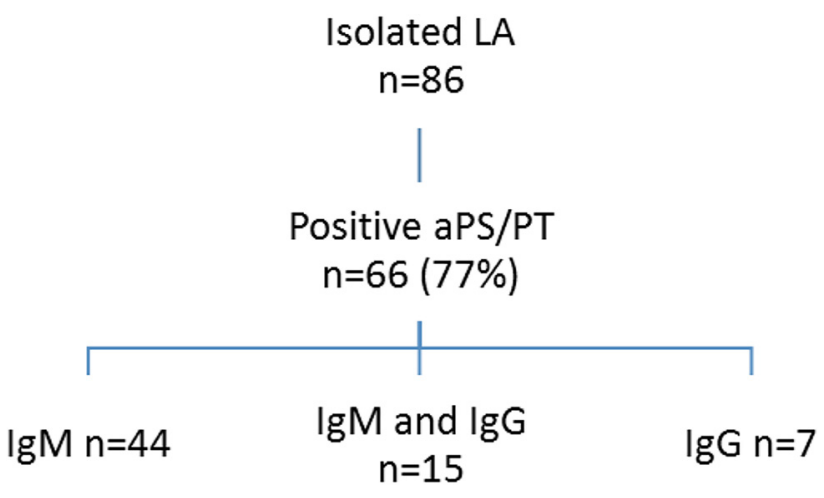

Figure 1: Of 436 non-anticoagulated LA positive individuals, 86 patients had isolated Lupus Anticogulant.

Of these, 66 were positive for the presence of anti phosphatidylserine/prothrombin antibodies. The predominant isotype was IgM.

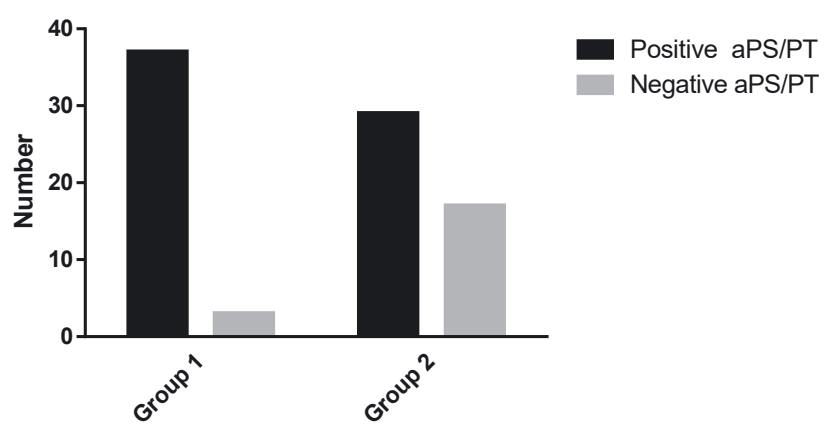

Figure 2: Patients with isolated LA were divided into two groups according to the way the diagnosis was made. In Group 1 both dRVVT and SCT assays were positive, while in Group 2 the diagnosis was made when only one test was positive. The presence of aPS/PT antibodies is significantly higher in patients of Group 1.

positive patients in which the percentage of patients with LA and dual positive test was $63 \%$ [8]. This difference might be explained by the presence in the cohort of triple positive patients in whom the potency of LA is higher than in patients with isolated LA [2]. A recent study reported a different figure of dual positive tests in LA detection as only $30 \%$ of patients were positive in both dRVVT and diluted aPTT (dAPTT) possibly in relation to the algorithm used in LA detection [11]. In the present study, we have shown that isolated LA is often associated with the presence of aPS/PT antibodies (77\%, mainly of IgM isotype) but not in all tested patients. According to guidelines, LA diagnosis is made when screening mixing and confirm studies are positive in at least one of the two tests chosen to detect the inhibitor. However, LA is much stronger in potency when both

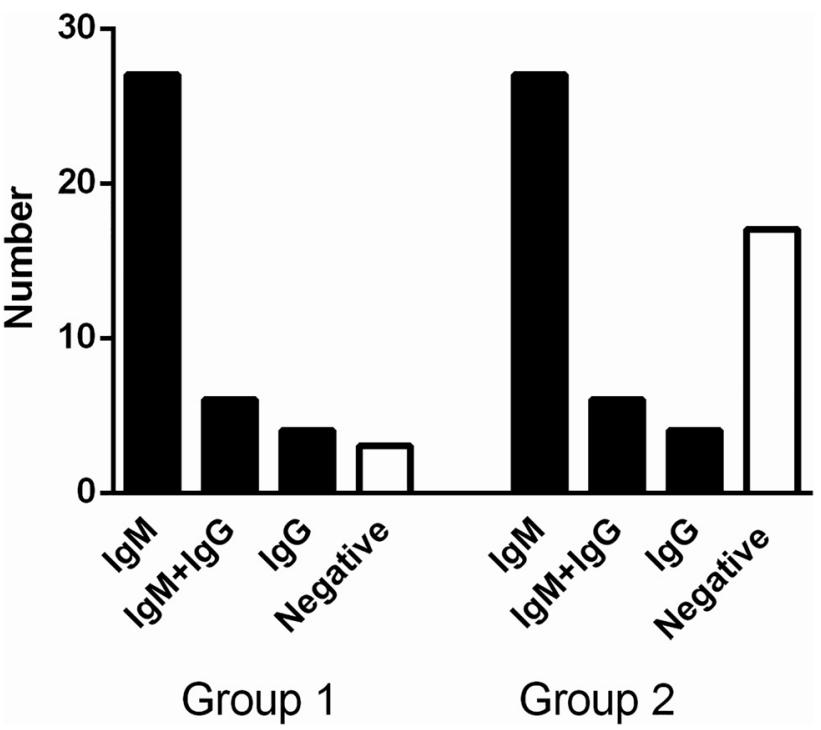

Figure 3: Number of LA positive individuals in Group 1 and Group 2 according to Immunoglobulin isotype.

\section{$\lg M$ aPS/PT}

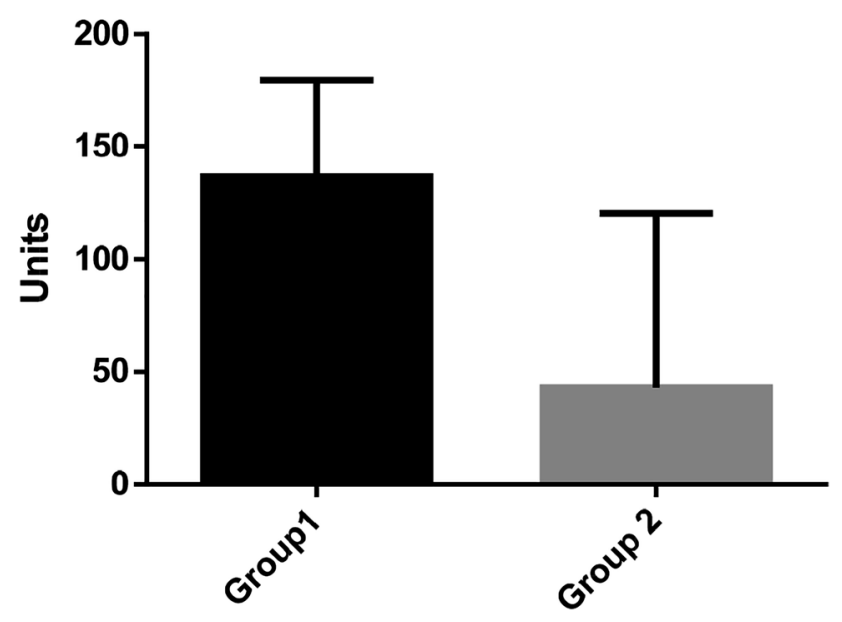

Figure 4: IgM was the predominant isotype in the studied cohort. Median IgM Units was significantly higher in Group 1.

dRVVT and aPTT-based tests are positive [8] and aPS/PT are responsible for LA in all the patient with strong isolated LA [2]. Therefore, we have raised the question of whether aPS/PT are present only in a subgroup of individuals with isolated LA in relation to the potency of the inhibitor. Thus, patients with isolated LA were divided in one group in which both dRVVT and SCT were positive and in the second one where either dRVVT or SCT were the only positive test. dRVVT is the most specific test in detecting significant LA $[12,13]$ and was the sole positive test in 15 of 46 patients of Group 2 while most of Group 2 patient were positive in SCT 


\section{IgG aPS/PT}

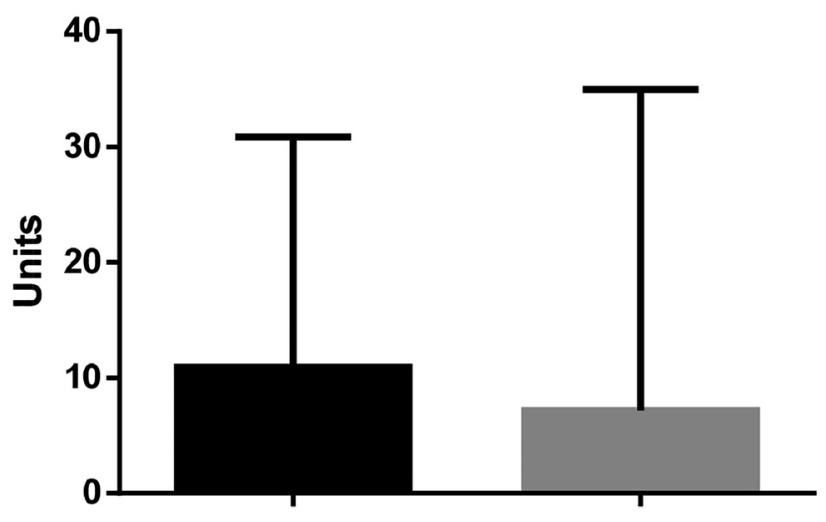

\section{Group $1 \quad$ Group 2}

Figure 5: Median IgG Units was not significantly different between Group 1 and Group 2.

only. These data may account for the significant discrepancy between aPS/PT positivity among the two groups. Indeed, the sole positive SCT might reflect the presence of mild or negligible LA or alternatively may account for false LA positive test. This point must be underlined as scientific reports on LA should always mention how the inhibitor was diagnosed. Moreover, this aspect may have clinical implication: LA positive for sole aPTT-based test may expose patients to an unjustified long-term oral anticoagulant treatment. Moreover, if isolated LA diagnosis is based only on positive aPTT and aPS/PT are negative either this is a mild or borderline positive LA or we are in the presence of a false positive LA or even LA is related to an unknown antibody.

Research funding: None declared.

Author contributions: V.P. conceived and planned the study and took the lead in writing the manuscript. M.T., E.B., E. P., M.G.C., C.Y.C, L.I. and G.D. performed the experiments and gave substantial contributions to acquisition and interpretation of data. All authors have accepted responsibility for the entire content of this manuscript and approved its submission.

Competing interests: V.P. received lecture fees from Werfen Group, Milan, Italy. All other authors state no conflict of interest.

Informed consent: After being tested for LA, patients gave their written informed consent to utilize their stored residual plasma in this study.

Ethical approval: The Institutional Review Board for observational studies and the Audit Committee of University of Padua Medical Centre approved the study design. The study was conducted in accordance with the principles of the Declaration of Helsinki.

\section{References}

1. Tektonidou MG, Andreoli L, Limper M, Amoura Z, Cervera R, Costedoat-Chalumeau N, et al. EULAR recommendations for the management of antiphospholipid syndrome in adults. Ann Rheum Dis 2019;78:1296-304.

2. Pengo V, Del Ross T, Ruffatti A, Bison E, Cattini MG, Pontara E, et al. Lupus anticoagulant identifies two distinct groups of patients with different antibody patterns. Thromb Res 2018;172: 172-8.

3. Pengo V, Testa S, Martinelli I, Ghirarduzzi A, Legnani C, Gresele P, et al. Incidence of a first thromboembolic event in carriers of isolated lupus anticoagulant. Thromb Res 2015;135:46-9.

4. Yin D, de Groot PG, Ninivaggi M, Devreese KMJ, de Laat B. Clinical relevance of isolated lupus anticoagulant positivity in patients with thrombotic antiphospholipid syndrome. Thromb Haemostasis 2020. https://doi.org/10.1055/a-1344-4271. [Online ahead of print].

5. Pengo V. Additional laboratory tests to improve on the diagnosis of antiphospholipid syndrome: response from Pengo. J Thromb Haemostasis 2020;18:3118-9.

6. Hoxha A, Banzato A, Ruffatti A, Pengo V. Detection of lupus anticoagulant in the era of direct oral anticoagulants. Autoimmun Rev 2017;16:173-8.

7. Pengo V, Tripodi A, Reber G, Rand JH, Ortel TL, Galli M, et al. Update of the guidelines for lupus anticoagulant detection. Subcommittee on lupus anticoagulant/antiphospholipid antibody of the scientific and standardisation committee of the international society on thrombosis and haemostasis. J Thromb Haemostasis 2009;7:1737-40.

8. Pengo V, Biasiolo A, Gresele P, Marongiu F, Erba N, Veschi F, et al. Survey of lupus anticoagulant diagnosis by central evaluation of positive plasma samples. J Thromb Haemostasis 2007;5:925-30.

9. Ruffatti A, Olivieri S, Tonello M, Bortolati M, Bison E, Salvan E, et al. Influence of different IgG anticardiolipin antibody cut-off values on antiphospholipid syndrome classification. J Thromb Haemostasis 2008;6:1693-6.

10. Devreese KMJ, Ortel TL, Pengo V, de Laat B. Subcommittee on Lupus Anticoagulant/Antiphospholipid A. Laboratory criteria for antiphospholipid syndrome: communication from the SSC of the ISTH. J Thromb Haemostasis 2018;16:809-13.

11. Moore GW, Maloney JC, de Jager N, Dunsmore CL, Gorman DK, Polgrean RF, et al. Application of different lupus anticoagulant diagnostic algorithms to the same assay data leads to interpretive discrepancies in some samples. Res Pract Thromb Haemostasis 2017;1:62-8.

12. Keeling D, Mackie I, Moore GW, Greer IA, Greaves M, British Committee for Standards in H. Guidelines on the investigation and management of antiphospholipid syndrome. $\mathrm{Br} J$ Haematol 2012;157:47-58.

13. Moore GW. Recent guidelines and recommendations for laboratory detection of lupus anticoagulants. Semin Thromb Hemost 2014;40:163-71. 